\title{
ON THE LAW OF EXCLUDED MIDDLE
}

\section{BY ALONZO CHURCH*}

1. Introduction. The purpose of this paper is to discuss the possibility of a system of logic in which the law of excluded middle is not assumed, and also to point out what seem to be errors in a recent papert in which the conclusion is reached that such a system of logic is self-contradictory.

The law of excluded middle is the logical principle in accordance with which every proposition is either true or false. This principle is used, in particular, whenever a proof is made by the method of reductio ad absurdum. And it is this principle, also, which enables us to say that the denial of the denial of a proposition is equivalent to the assertion of the proposition.

The simplest alternative to the inclusion of the law of excluded middle among the principles of logic is its bare omission without assertion of any contrary principle. The effect of such an omission is, of course, to reduce the number of theorems which can be proved and also to render of interest certain theorems otherwise vacuous. We cannot derive theorems which contradict theorems obtained with the aid of the law of excluded middle unless we make some assertion of a principle which contradicts the law of excluded middle.

It is not possible, as an alternative to the law of excluded middle, to assert that some proposition is neither true nor false, because by so doing not only the law of excluded middle would be denied but also the law of contradiction. In fact, to assert that a proposition $p$ is not true and is also not false is to assert at once not- $p$ and not-(not-p) and consequently to assert that not- $p$ is both true and false.

* National Research Fellow in Mathematics.

$\dagger$ M. Barzin and A. Errera, Sur la logique de M. Brouwer, Académie Royale de Belgique, Bulletins de la Classe des Sciences, (5), vol. 13 (1927), pp. 56-71. 
It may be, however, that, by introducing the middle ground between true and false as an undefined term, let us say "tiers" (adopting from the French the word used by Barzin and Errera), and making an appropriate set of assumptions about the existence and properties of tiers propositions, we can produce a system of logic which is consistent with itself but which becomes inconsistent if the law of excluded middle be added.

2. The Position of L. E. J. Brouwer. L. E. J. Brouwer proposes that the law of excluded middle should not be regarded as an admissible logical principle, ${ }^{*}$ and expresses, as a basis for his proposal, doubts concerning the truth of this law. He says, for example, that the law of excluded middle has been extended to the mathematics of infinite classes by an unjustified analogy with that of finite classes. He says also that to assert the law of excluded middle is equivalent to asserting the doubtful proposition that every proposed theorem can be either proved or disproved if the proper method be found. The latter point depends, of course, on identifying the truth of a proposition with the possibility of proving the proposition. But it seems more in accord with our usual ideas to think of truth as a property of a proposition independent of our ability to prove it. Consequently we prefer to take the truth of a proposition merely as an undefined term subject to certain postulates, among them, if we choose to include it, the law of excluded middle.

In connection with geometry and other branches of mathematics it is commonly recognized that it is meaningless to ask about the absolute truth of a postulate and that the choice between one of two contrary postulates must be made on the basis of simplicity and serviceability. It seems reasonable to recognize the same thing with regard to the postulates of logic, in particular the law of excluded middle, and to say on this basis that it is meaningless to ask about the truth of the law of excluded middle.

* See, for example, Intuitionistische Mengenlehre, Jahresbericht der Deutschen Mathematiker-Vereinigung, vol. 28 (1919), pp. 203-208. 
Taking this point of view, we may accept a system of logic in which the law of excluded middle is assumed, a system in which the law of excluded middle is omitted without making a contrary assumption, and a system which contains assumptions not in accord with the law of excluded middle as all three equally admissible, unless one of them can be shown to lead to a contradiction. If we had to choose among these systems of logic, we could choose the one most serviceable for our purpose, and we might conceivably make different choices for different purposes.

3. Barzin and Errera's Paper. Barzin and Errera, however, reach the conclusion (loc. cit.) that the system of logic proposed by Brouwer leads to contradiction. This conclusion we believe to be erroneous, for the following reasons.

The method of the argument is the method of reductio ad absurdum. It is assumed that if the law of excluded middle is not accepted then it must be explicitly denied by asserting the existence of tiers propositions, and on this basis contradictory results are obtained. This argument is clearly not effective against one who merely omits the law of excluded middle from his system of logic without assuming any contrary principle, because the insistence that one who refuses to accept a proposition must deny it can be justified only by an appeal to the law of excluded middle, the very principle in doubt. The method of reductio ad absurdum, in fact, necessarily employs the law of excluded middle and cannot be used against one who does not admit this law.

The argument of Barzin and Errera would not be effective, however, even against one who asserted the existence of tiers propositions, because in the course of the argument it is necessary to assume what the authors name the principle of excluded fourth, that every proposition is either true, false, or tiers, a principle which seems to be a restricted form of the law of excluded middle. This assumption is defended on the basis of the definition of a tiers proposition as one which is neither true nor false, but this definition, as pointed out above, is not consistent with itself and consequently 
cannot be used. If the concept tiers is introduced at all, it must be as an undefined term.

If we admit the concept tiers and the principle of excluded fourth, the argument of Barzin and Errera is then quite correct. They show that " $p$ is tiers" together with " $p$ obeys the principle of excluded fourth" implies a contradiction; hence that $p$ is not tiers; and hence, by an appeal to the principle of excluded fourth, that $p$ is either true or false. But one who asserts the existence of tiers propositions need not assert the principle of excluded fourth; and one who merely omits the law of excluded middle without introducing the concept tiers certainly could not assert the principle of excluded fourth.

It would not be permissible to say that the word tiers is to cover all possibilities other than true and false, however many such possibilities there may be. This language is, in fact, equivalent to defining tiers to mean neither true nor false, and is open to the same objections. But the following objection could also be raised. Using $p^{\prime}$ to stand for " $p$ is tiers," it seems likely that $\left(p^{\prime}\right)^{\prime}$, or $p^{\prime \prime}$, would constitute a fourth possibility, $\left(p^{\prime \prime}\right)^{\prime}$ a fifth possibility, and so on. Uniting these possibilities into one under a new name, let us say $p^{*}$, we then have to reckon with the likelihood that $\left(p^{*}\right)^{\prime}$ gives still another possibility which we may designate by the transfinite ordinal $\omega$. And after this we have to reckon with an $(\omega+1)$ th possibility, and so on, so that ultimately we reach any given transfinite ordinal whatever. Any attempt to unite all these possibilities into one must be regarded as extremely doubtful, because of its connection with Burali-Forti's paradox $\dagger$ concerning the ordinal number of the sequence of all transfinite ordinals. This paradox, in fact, compels us to regard as illegitimate the consideration of this sequence as a whole.

HARVARD UNIVERSITY

† C. Burali-Forti, Una questione sui numeri transfiniti, Rendiconti del Circolo Matematico di Palermo, vol. 11 (1897), pp. 154-164. Whitehead and Russell, Principia Mathematica, vol. III, pp. 73-75. 